\title{
Bürgerbeteiligung oder "Stimme des Volkes«? Deliberative Partizipationsformen in verfassungsändernden Prozessen auf gesamtstaatlicher Ebene
}

Jens Woelk"

\section{Der Rabmen: Partizipation an Änderungen der geltenden Verfassung}

Partizipative Prozesse deliberativer Art werden seit einigen Jahren auch im Kontext von Verfassungsänderungen angewendet. Entsprechend ihrer Ursprünge in Verwaltungsverfahren, wo partizipative Elemente Bürger stärker in die Governance von Gemeingütern einbinden sollen, sind Formen deliberativer Bürgerbeteiligungsprozesse auf verfassungsrechtlicher Ebene vor allem im Rahmen von Verfassungsänderungen eingesetzt worden. Also zur Veränderung geltender Verfassungen und als Ergänzung repräsentativ-demokratischer Elemente und nicht zur Verfassungsgebung im Sinne der Ablösung einer bestehenden bzw. der Erarbeitung einer komplett neuen Verfassung. Die Verfassungsgebung ist typischerweise Aufgabe einer gewählten, verfassungsgebenden Versammlung, als Ausdruck der Volkssouveränität, welche dem gesamten Verfassungssystem Legitimation und Legitimität verleiht.

Diese konzeptionelle Abgrenzung ist auch inhaltliche Begrenzung: sie legt den Rahmen für den Einsatz partizipativer Elemente in Verfassungsänderungsverfahren fest. Inhaltlich geht es nicht um eine Revision der Verfassungsgrundlagen oder der obersten Verfassungsgrundsätze (»Bauprinzipien« der Verfassung), sondern eher um »Wartungsarbeiten« am von den Verfassungsgrundsätzen festgelegten Rahmen, ${ }^{1}$ d.h. um die Anpassung oder Aktualisierung (»Nachführung«) der Verfassung in einzelnen wichtigen Punkten, ${ }^{2}$ aber durchaus auch um größere Reformen auf einzelnen Gebieten.

* Der Beitrag fußt auf einem Vortrag, der im Rahmen der Tagung »Governance auf dem Prüfstand: Direkte und deliberative Demokratie in Europa« vom 26.-27. Oktober 2017 in Bozen gehalten wurde.

1 Francesco Palermo (Hrsg.), La manutenzione costituzionale (Cedam, 2007).

2 »Nachführung « ist der für die Totalrevision der schweizerischen Bundesverfassung 1999 gewählte Begriff, der treffend die Intention beschreibt, den Text der Verfas- 
Selbstverständlich stellt sich die Frage nach der Abgrenzung zwischen einzelnen Verfassungsänderungen (insbesondere, wenn gleichzeitig mehrere erarbeitet und vorgenommen werden sollen) und einer Gesamtänderung der Verfassung. In qualitativer Hinsicht ist für eine derartige Grenzziehung entscheidend, dass oberste Verfassungsgrundätze nicht berührt oder in Frage gestellt werden, die etwa durch Ewigkeitsklauseln (z.B. Art. 79 Abs. 3 Grundgesetz - GG), oder als »Baugesetze« (im österreichischen Verfassungsrecht) besonderen Schutz genießen. Der Verfassungskern bleibt also unverändert erhalten. Obwohl die Abgrenzung zwischen Verfassungsänderung und Verfassungsgebung als klare Unterscheidung erscheint, kann sie bei einer quantitativen Betrachtungsweise durchaus relativiert werden, wenn die Zahl der Änderungen in verschiedenen Bereichen so groß ist, dass die Summe der Änderungen Auswirkungen auf die Gesamtsubstanz der Verfassung zeitigt.

Das Beispiel Italiens zeigt dies deutlich: Während sich die sog. Föderalisierungsreform von 2001 auf den V. Titel des II. Teils der italienischen Verfassung (itVerf) und das Verhältnis Staat-Regionen beschränkte, enthielten sowohl der Berlusconi-Reformvorschlag (2006) und der Renzi/Boschi-Reformvorschlag (2017) Änderungen von mehr als 50 Artikeln. ${ }^{3}$ Diese letztlich im Referendum abgelehnten Änderungsvorhaben waren zwar auf den II. Teil der Verfassung beschränkt (Staatsorganisation), hätten jedoch das Gesamtbild der italienischen Republik sowohl im Hinblick auf die Regierungsform als auch in Bezug auf die Organisation der Gebietsverwaltung grundlegend verändert. ${ }^{4}$

Im Folgenden werden, nach einer kurzen Betrachtung zur pluralistischen Demokratie und zu den Entscheidungsprozessen und Änderungsverfahren auf verfassungsrechtlicher Ebene, verschiedene Anwendungsfälle partizipativer Demokratie im verfassungsrechtlichen Bereich vorgestellt

sung wieder mit ihrer Substanz in Übereinstimmung zu bringen, in dem geänderte rechtliche, soziale und internationale Rahmenbedingungen berücksichtigt werden.

3 Zu den Verfassungsreformvorschlägen Berlusconi (2006) und Renzi/Boschi (2016) vgl. die Beiträge in EZFF (Hrsg.), Jahrbuch des Föderalismus 2006 (Nomos, 2006) und EZFF (Hrsg.), Jahrbuch des Föderalismus 2016 (Nomos, 2016) der entsprechenden Jahre sowie Elisabeth Alber »Regionalstaat Italien - Reformblockaden und Perspektiven«, (2018) 1 Gesellschaft - Wirtschaft - Politik $75 \mathrm{ff}$.

4 Der (politisch) allgemein als änderungsfest betrachtete erste Teil der italienischen Verfassung beinhaltet neben den Staatsgrundsätzen (Art. 1-12 itVerf) die Grundrechtsgarantien, während der zweite Teil die Staatsorganisation (Verfassungsorgane, Staat-Regionen, Justiz, etc.) beinhaltet und in einzelnen Punkten oder Abschnitten schon häufiger geändert wurde. 
und untersucht. Grundannahme ist, dass deliberative und partizipative Elemente entsprechend ihrer Natur auch im Verfassungsbereich vorwiegend als komplementäre Elemente eingesetzt werden, zur Vorbereitung wichtiger Änderungen durch vertiefte Diskussion und zur Steigerung der Legitimität der Entscheidung, regelmäßig im Vorfeld formeller Verfassungsänderungsverfahren.

Es werden Beispiele für die (gesamt)staatliche Ebene bzw. Bundesebene gegenübergestellt und kurz diskutiert. ${ }^{5}$ Das Hauptaugenmerk gilt dabei neben den Gründen, die zur Anwendung deliberativer und partizipativer Elemente geführt haben, dem Verhältnis zum ordentlichen, für Verfassungsänderungen vorgesehenen Verfahren und der Art und Weise der Organisation von Bürgerbeteiligungsprozessen (Auswahl der Teilnehmer, Arbeitsmethoden, Verbindlichkeit der Ergebnisse). Entsprechend wird im Folgenden die vergleichende Untersuchung der einzelnen Fälle strukutiert.

\section{Pluralistische Gesellschaften zwischen Populismus und nachhaltigen Lösungen}

Ausgangspunkt aller Betrachtungen ist der Pluralismus, der die heutigen demokratischen Gesellschaften kennzeichnet und der in ihren Verfassungen sowohl als objektiver Wert als auch durch den Schutz individueller Grundrechte garantiert wird. ${ }^{6}$ Pluralismus führt notwendigerweise zu größerer Komplexität, da verschiedene und teilweise gegenläufige Wertvorstellungen und Rechte in einem gesamtgesellschaftlichen Rahmen integriert und zu einem Ausgleich gebracht werden müssen. Dies geschieht täglich durch alle Gewalten: den Gesetzgeber, die Verwaltungsbehörden und durch die Gerichte. Eine besondere Rolle spielen Verfassungsgerich-

5 Der Beitrag von Elisabeth Alber in diesem Band geht auf Beispiele auf der subnationalen, gliedstaatlichen Ebene ein. Teija Tiilikainen befasst sich mit dem EU-Verfassungskonvent.

6 In der Tradition der Aufklärung und versinnbildlicht durch die bekannte, treffende Formulierung des "pursuit of happiness « (Strebens nach Glück), als dem Inbegriff persönlicher, individueller Autonomie, welche es dem Individuum ermöglicht, sich selbst nach seinen eigenen Vorstellungen zu verwirklichen. Nicht das Glück als solches ist garantiert (da dieses von den jeweiligen individuellen Vorstellungen abhängt), sondern die Freiheit, dieses persönlich und individuell definierte Glück anzustreben. Gesamtgesellschaftlich betrachtet führen die Unterschiede in den Vorstellungen zu einem breiten Spektrum möglicher Glücksvorstellungen, welche Ausdruck des - verfassungsrechtlich geschützten - Pluralismus sind. 
te, welche die von diesen Gewalten getroffenen Entscheidungen auf ihre Vereinbarkeit mit dem verfassungsrechtlichen Rahmen überprüfen, was insbesondere für Minderheiten in pluralistischen Gesellschaften eine unverzichtbare Garantie ist. ${ }^{7}$

Bessere Ausbildung, ein größeres demokratisches Bewusstsein und ständig und überall verfügbare Information sollten zu einem größeren Interesse an wichtigen Entscheidungen führen. Eine verbreitete Unzufriedenheit mit Politik an sich, mit der Komplexität vieler wichtiger Fragen und das Gefühl eines großen Abstandes zwischen den Bürgern und »den Institutionen « sind von populistischen Bewegungen ausgenutzt worden, welche die repräsentative Demokratie in Frage stellen. Diese Bewegungen appellieren typischerweise direkt an »das Volk«, wobei Führerpersönlichkeiten (»Leader«) und plebiszitäre Elemente eine wichtige Rolle spielen. Komplexe Probleme werden in der Debatte in der Regel verengt auf binäre, alternative Fragestellungen (»Ja« und »Nein«; »dafür« und »dagegen«), über welche »das Volk« mit Mehrheit entscheiden soll (wobei die - richtige - Antwort durch die Alternative »für uns oder gegen uns" bereits häufig in der Fragestellung suggeriert wird). Direkte Demokratie und damit das Mehrheitsprinzip sollen stärker eingesetzt werden, ohne Rücksicht auf Grundwerte, Rechte anderer und die Komplexität vieler Sachfragen in modernen, pluralistischen und vernetzten Gesellschaften. Repräsentative Entscheidungsprozesse und ihre Filter- und Repräsentationsfunktion werden demgegenüber als langsam, ineffizient und als von »herrschenden Eliten" kontrolliert kritisiert. Auch die Krise der politischen Parteien in ihrer Vermittlerfunktion zwischen Institutionen und Wählern ist Ausdruck dieser Veränderungen.

Es stellt sich daher generell die Frage, wie in Entscheidungsverfahren Komplexität und Pluralismus berücksichtigt und garantiert werden können und gleichzeitig die Akzeptanz gestärkt werden kann. ${ }^{8}$ Dies gilt umso

7 Die gerichtliche Überprüfung demokratischer Mehrheitsentscheidungen ist zur Verteidigung gegen solche Mehrheitsentscheidungen notwendig, welche den betroffenen Angehörigen von politischen, sozialen, religiösen oder anderen anerkannten Minderheiten trotz pluralistischer Wertentscheidungen in der Verfassung keinen oder zu wenig Raum für autonome Entscheidungen lassen; sie dienen also der Grenzziehung zwischen Autonomie und Integration (vgl. Joseph Marko, Autonomie und Integration (Böhlau, 1995) im konkreten Einzelfall und gleichzeitig der dynamischen Weiterentwicklung und Anpassung des gesamten Wertesystems durch Auslegung der allgemeinen Grundsätze und Grundrechte.

8 Vgl. bspw. Ralf Dahrendorf, Die Krisen der Demokratie (C.H. Beck, 2002); Donatella della Porta, »Democrazia: sfide e opportunità», (2010) 2 Rivista Italiana di Scienza Politica 175; Donatella della Porta, Can Democracy be Saved? Participation, 
mehr auf verfassungsrechtlicher Ebene, bei der Anpassung und Änderung der demokratischen Grundregeln.

Die Grundsätze der deliberativen Demokratie, welche sich in den letzten Jahrzehnten auf verwaltungsrechtlicher Ebene in verschiedensten Formen stärkerer und direkter Beteiligung von Bürgern und Interessenvertretungen herausgebildet und konsoliert haben, sowie eine direkte Beteiligung und Partizipation der Bürger via Internet eröffnen auch im verfassungsrechtlichen Bereich neue Möglichkeiten.

Die Suche nach Alternativen, welche zusätzliche Partizipationsmöglichkeiten bieten, wird dadurch entscheidend bestimmt, dass Änderungen der Verfassung regelmäßig in besonderen Verfahren und mit besonderen, übergreifenden Mehrheiten erfolgen müssen. Dadurch sollen Grundrechte und Verfassungsgrundsätze vor übereilten Entscheidungen geschützt und der Pluralismus als Grundlage des gesellschaftlichen Zusammenlebens garantiert werden. Trotz des Demokratieprinzips werden Mehrheitsentscheidungen in diesen Bereichen zum Schutze von Grundwerten und Grundrechten durch besondere Verfahren und Mehrheiten bewußt erschwert oder ihnen sogar absolute Grenzen gesetzt (absolute Garantien, bspw. republikanische Staatsform in Italien, Art. 139 itVerf, oder »Ewigkeitsklausel«, Art. 79 Abs. 3 GG).

Die für Verfassungsänderungen typischen besonderen Verfahren basieren zwar zumeist auf dem ordentlichen Gesetzgebungsverfahren, weisen aber in der Regel zusätzliche Verfahrenserfordernisse auf, z.B. qualifizierte Mehrheiten, Fristen, wiederholte Abstimmungen, etc. Wegen der Wichtigkeit der allgemeinen Akzeptanz der Grundnormen in der pluralistischen Gesellschaft, sind grundlegende Entscheidungen besonders zu überdenken; die genannten Erschwernisse haben daher eine Warnfunktion. Auch die repräsentative Demokratie wird teilweise einbezogen, wie etwa durch die Erfordernisse von Auflösung und Neuwahlen sowie der notwendigen Bestätigung des Reformvorhabens durch das neugewählte Parlament, die in einigen Verfassungsordnungen vorgesehen sind. ${ }^{9}$ Dies soll dem Volk effektive Mitwirkung und Kontrolle ermöglichen - durch Wiederwahl oder Abwahl der Reformmehrheit. Nicht selten findet sich aus diesem Grund eine Kombination von Verfahrenserschwernissen im Parlament mit einem Referendum als Abschluss des Verfahrens: die direkte Demokratie

Deliberation and Social Movements (Polity Press, 2013); Angelika Vetter und Uwe Remer-Bollow, Bürger und Beteiligung in der Demokratie (VS Springer, 2017).

9 So muss z.B. in Belgien nach Art. 195 belgischer Verfassung, das Parlament nach einer Verfassungsänderung aufgelöst werden, damit nach Wahlen das Parlament in neuer Zusammensetzung diese ratifizieren kann. 
dient hier der Bestätigung der Parlamentsinitiative. ${ }^{10}$ Beispiele für eine derartige Kombination sind Italien und Österreich. Ein Ausnahmefall ist Deutschland, wo lediglich 2/3-Mehrheiten in Bundestag und Bundesrat vorgesehen sind und eine Beteiligung der Wähler an Verfassungsänderungen entsprechend grundsätzlich nicht vorgesehen ist.

In herkömmlichen Verfassungsänderungsverfahren finden sich - außer den genannten Volksentscheiden, die jedoch der abschließenden Bestätigung einer Reform dienen - in der Regel keine Möglichkeiten der Bürgerbeteiligung. Es stellt sich damit die Frage, wie und wo derartige deliberative Partizipationsformen durchgeführt werden können, ohne dass dies die Rechtmäßigkeit der Verfahren berührt. Einige Verfassungsordnungen haben deswegen partizipative Formen außerhalb bzw. vor Beginn der eigentlichen formellen Verfahren eingeführt. Diese haben insoweit eine vorbereitende bzw. beratende Funktion, wobei der gegenüber den formellen Verfahren erweiterte Kreis der Teilnehmer intensive Abwägung und Erörterung aller Argumente und damit Nachhaltigkeit garantieren soll.

\section{Der EU-Verfassungskonvent: Sonderfall und Vorläufer}

Trotz der Tatsache, dass die Europäische Union nach wie vor eine internationale Organisation ist, die auf völkerrechtlichen Verträgen zwischen den Mitgliedstaaten gründet, wurde im ersten Jahrzehnt des Jahrhunderts versucht, eine Verfassungsdebatte zu führen, mit dem Ziel die Verfassungsqualität vieler Vorschriften der EU Verträge stärker im Bewußtsein der Bevölkerungen zu verankern. Im Mittelpunkt der Diskussion stand das Ziel vertiefter Integration sowie die Erarbeitung eines »Verfassungsvertrages«,

10 Art. 138 itVerf schreibt zwei Abstimmungen in beiden Häusern vor, im Abstand von drei Monaten. Bei absoluter Mehrheit in der zweiten Abstimmung kann ein Referendum beantragt werden (von 1/5 der Abgeordneten oder Senatoren, 500.000 Wählern, oder fünf Regionalräten); sollte das Parlament die Reform mit einer 2/3 Mehrheit verabschieden, ist kein Referendum notwendig. In Österreich ist eine 2/3-Mehrheit im Nationalrat (bei Anwesenheit von mindestens der Hälfte der Mitglieder) zur Verabschiedung von Änderungen der Bundesverfassung oder gesetzlicher Vorschriften im Verfassungsrang erforderlich, Art. 44 Abs. 1 B-VG. Der Bundesrat muss dem ebenfalls mit 2/3-Mehrheit zustimmen, sollten die Änderungen die Kompetenzen der Bundesländer betreffen, Art. 44 Abs. 2 B-VG. Ein Referendum ist bei Totalrevision der Verfassung zwingend vorgeschrieben (wenn deren Substanz, die »Baugesetze« von einer Änderung berührt werden); bei anderen Änderungen kann ein Referendum von einem Drittel der Nationalratsabgeordneten oder der Bundesratsmitglieder verlangt werden, Art. 44 Abs. 3 B-VG. 
der zwar weiter völkerrechtliche Rechtsquelle sein sollte, inhaltlich aber wesentlich stärker einer staatlichen Verfassung ähneln sollte.

Die Verfassungsdiskussion war auch von der damals bevorstehenden großen EU-Osterweiterung angestoßen, da im Hinblick auf diese Erweiterung um 10 neue Mitglieder sichere Integrationsgrundlagen geschaffen werden sollten. Die Beratungen im Rahmen der Regierungskoferenz der Mitgliedstaaten sollten durch partizipative Elemente ergänzt werden, sowohl um den besonderen Charakter der geplanten Änderungen zu betonen, als auch um deren Legitimation zu erhöhen.

Der Prozess konnte auf die Erfahrungen mit der EU GrunderechteCharta bauen. Zu ihrer Erarbeitung wurde auf dem Kölner Gipfel 1999 zunächst eine ad hoc-Versammlung einberufen, welche sich (in Anspielung auf den US Verfassungskonvent von Philadelphia von 1787) selbst zum "Konvent« erklärte. An diesem Konvent nahmen 15 Vertreter der Staatsund Regierungschefs der Mitgliedstaaten teil, 30 Vertreter der nationalen Parlamente, 16 des Europaparlaments, ein Vertreter der Kommission sowie verschiedene Beobachter (des Europäischen Gerichtshofes, des Europarates und anderer Organisationen).

Nach dieser ersten, weitgehend positiv bewerteten Erfahrung beschloss der Europäische Rat in Laeken einen weiteren »Konvent für die Zukunft Europas« einzuberufen und ihm die Aufgabe zu übertragen, einen Entwurf für einen Verfassungsvertrag für die Europäische Union auszuarbeiten. Nach dem Vorbild des Grundrechtecharta-Konvents sollten 105 Abgeordnete nationaler Parlamente (der Mitgliedstaaten und der damaligen Kandidaten für einen Beitritt), Abgeordnete des Europaparlaments, der Europäischen Kommission sowie Vertreter der Staats- und Regierungschefs in öffentlichen Sitzungen arbeiten. Die partizipative Methode sollte ein offenes Diskussionsforum schaffen, im Gegensatz zur Regierungskonferenz, in der die Beratungen hinter verschlossenen Türen stattfinden. ${ }^{11}$

Der durch den Konvent unter dem Präsidenten Giscard d'Estaing erarbeitete Verfassungsvertrag wurde anschließend durch die Regierungskonferenz verabschiedet, trat jedoch nie in Kraft, da die Ratifizierungsphase nach den negativen Referenda in Frankreich und den Niederlanden schließlich vorzeitig abgebrochen wurde. Trotzdem wurden die Erfahrungen mit dem Konvent weithin positiv gewertet. Nach einer zweijährigen Reflektionsphase wurden wesentliche Inhalte des gescheiterten Verfas-

11 Der EU Verfassungskonvent wird im Beitrag von Teija Tiilikainen in diesem Band ausführlich untersucht und diskutiert. In diesem Beitrag geht es darum, seine Vorbildwirkung für nationale Verfassungsreformprozesse zu unterstreichen. 
sungsvertrages in den Vertrag von Lissabon übernommen, der am 1. Dezember 2009 in Kraft trat. Zu den wesentlichen Neuerungen gehört die Neuregelung des Vertragsänderungsverfahrens: der Konvent wird nun als - vorgeschalteter - Bestandteil des förmlichen Vertragsänderungsverfahrens ausdrücklich anerkannt (Art. 48.1 EUV).

Die Diskussion der Konventsmethode und der konkreten Erfahrungen mit dem EU Konvent haben die Verfassungsreformdiskussionen in mehreren Mitgliedstaaten nachhaltig beeinflußt.

\section{Der Österreich-Konvent: ein Experiment mit Folgen}

Von Juni 2003 bis Januar 2005 wurde in Österreich ein intensiver Diskussionsprozess über eine Staats- und Verfassungsreform durchgeführt. Ein eigens dafür einberufener »Österreichkonvent « erarbeitete zahlreiche Reformvorschläge und legte einen umfassenden Abschlussbericht vor. ${ }^{12}$ Direkt gewählte Mandatare der Landtage bzw. des Nationalrates waren im Österreichkonvent ebenso vertreten wie die Selbstverwaltungskörperschaften, insbesondere Gemeinden und Städte.

Aus politischen Gründen wurden die Vorschläge jedoch schließlich nicht in einer einzigen großen Reform verabschiedet; stattdessen sollten sie allmählich und schrittweise umgesetzt werden. ${ }^{13}$ Nach Abschluss der Arbeiten übermittelte der Bundeskanzler den »Bericht des Österreich-Konvents« dem Nationalrat, der die Ergebnisse des Konvents bis Juli 2006 in einem »Besonderen Ausschuss zur Vorberatung des Berichts des Österreich-Konvents« behandelte. Im Frühjahr 2007 wurde dann durch die neue

12 Vgl. zu den einzelnen Verfahrensabschnitten und für den Abschlussbericht das Portal des Verfassungskonvents (www.konvent.gv.at/K/Willkommen_Portal.shtm 1); hier findet sich auch eine umfassende Auswahlbibliographie zu den Arbeiten des Konvents und ihrer Rezeption.

13 Ludwig Adamovich, »Nach dem Österreich-Konvent; Bereinigung oder grundlegende Änderung der Verfassung? «, in Bundesministerium für Inneres (Hrsg.), Verfassung - Reform - Rechtsschutz. 3. Rechtsschutztag des Bundesministeriums für Inneres (NWV, 2006), 27ff; Barbara Blümel, Österreich-Konvent - Die Umsetzung der Verfassungsrevision (Demokratiezentrum Wien, 2004); Emil Brix, Jürgen Nautz und Klaus Poier (Hrsg.), Die österreichische Verfassungsdiskussion und die Zivilgesellschaft (Passagen 2006); Peter Bußjäger, Klippen einer Föderalismusreform - Die Inszenierung Österreich-Konvent zwischen Innovationsresistenz und Neojosephinismus (Institut für Föderalismus, 2005); Anna Gamper, »The Austrian Constitutional Convention: Continuing the Path to Reform the Federal State? «, (2006) 2 Revista d'Estudis Autonòmics I Federals 9. 
Bundesregierung eine Expertengruppe beim Bundeskanzleramt gebildet, deren Vorschläge zur Grundlage für Parlamentsbeschlüsse über ein »Demokratiepaket«, die Bereinigung des Bundesverfassungsrechts, die Neuregelung weisungsfreier Behörden und die Einrichtung eines Asylpakets wurden. Auch konnte ein neues Haushaltsrecht beschlossen werden, das seine Grundlagen in den Beratungen des Österreich-Konvents hatte.

Weitere Vorschläge des Österreich-Konvents und der Expertengruppe, insbesondere zur Bundesstaatsreform, wurden jedoch nicht weiter behandelt. ${ }^{14}$ Dagegen wurden in der folgenden Legislaturperiode (ab 2008) weitere Vorschläge zu Reformen in den Bereichen des Wahlrechts und der Verwaltungsorganisation umgesetzt. Außerdem wurde 2012 die Einrichtung von Verwaltungsgerichten im Parlament verabschiedet: seit 1. Januar 2014 arbeiten in Österreich nun neun Landesverwaltungsgerichte, ein Bundesverwaltungsgericht und ein Bundesfinanzgericht. Auch diese weitreichende Reform geht auf die Beratungen und Vorschläge des ÖsterreichKonvents zurück.

In der Folge wurde 2014 eine parlamentarische Enquete-Kommission zur Aufwertung direktdemokratischer Instrumente eingerichtet, der neben 18 Abgeordneten als stimmberechtigte Mitglieder neun weitere ständige Mitglieder (Bundesratsmitglieder und Experten) ohne Stimmrecht angehörten. Darüber hinaus nahmen jedoch auch acht, in einem Losverfahren ermittelte Bürger mit Rederecht an den Sitzungen teil. Trotz dieser interessanten Neuerung gab es nach den Beratungen der Enquetekommisison jedoch keinen fraktionsübergreifenden Konsens im Parlament, wie die direkte Demokratie in Österreich weiterentwickelt werden soll.

Das Ergebnis dieser Versuche, Formen deliberativer Demokratie vor grundlegenden Reformen in die formellen Verfahren einzuführen, kann als verhalten positiv gewertet werden. Der Österreich-Konvent hat zwar selbst unmittelbar keine umfassende Reform bewirkt, jedoch haben seine Beratungen in der Folge einzelne, wichtige Reformschritte vorbereitet und geprägt. Dagegen haben die Versuche, durch Los bestimmte Bürger direkt in die parlamentarischen Beratungen einzubinden aufgrund des Übergewichts der Parlamentarier und der dominanten repräsentativ-demokratischen Logik weniger überzeugt; sie gingen in der parteipolitisch geprägten Dynamik des Parlamentsbetriebes unter. Parallel wurden Bürgerdialoge

14 Christoph Konrath, »Dann bleibt es ebenso: Föderalismus und Kompetenzverteilung als Themen des Österreich-Konvents«, (2005) 34 Österreichische Zeitschrift für Politikwissenschaft 351; Ewald Wiederin, »Bundesstaatsreform in Österreich", in Detlef Merten (Hrsg.), Die Zukunft des Föderalismus in Deutschland und Europa (Duncker \& Humblot, 2007) 87ff. 
organisiert. Auch diese waren jedoch alles andere als wirksam im Hinblick auf die tatsächlich getroffenen Entscheidungen und das Feedback von Seiten der Politik; sie sind aber als innovative Partizipationsform ein interessantes Detail. ${ }^{15}$ Im Oktober 2018 hat die österreichische Regierung erneut Themen der Diskussionen aufgegriffen und weitreichende Vorschläge für eine Föderalismusreform vorgestellt. ${ }^{16}$

\section{Föderalismusreform in Deutschland: Auslagerung statt partizipativer Modelle}

In Deutschland gibt es auf der Bundesebene keine rechtlichen Grundlagen mit direkter oder partizipativer Demokratie und daher keine Erfahrungswerte, während direktdemokratische und deliberative Partizipationsinstrumente auf Länderebene und in den Gemeinden immer stärker genutzt werden. Vor 15 Jahren hatte die Diskussion um eine Reform des Grundgesetzes an Intensität deutlich zugenommen und konzentrierte sich, in verfassungsrechtlicher Hinsicht, auf einen zentralen Aspekt: das bundesstaatliche System. Es wurden vor allem mehr Wettbewerb gefordert und die zentrale Rolle des Bundesrates in Frage gestellt, als Hindernis in Entscheidungsprozessen. Dabei ist der Bundesrat die institutionelle Klammer des Demokratie- und des Bundesstaatsprinzips, welche beide für die deutsche Verfassungsordnung wesentlich sind. Er vereinigt beide Prinzipien institutionell durch Mitwirkung der Länder im Gesetzgebungsverfahren, mit Einspruchs- und Zustimmungsgesetzen. Wegen dieser Mitwirkung und der damit verbundenen Verlangsamung und einigen Blockaden wurde der Bundesrat als Ursache für den »Reformstau« beschuldigt. Dem sollte eine mögliche Reform im Sinne einer Entflechtung des bundesstaatlichen Systems und einer Reduzierung der Partizipationsrechte des Bundesrates abhelfen.

Für jede Änderung des Grundgesetzes sind aber Zweidrittelmehrheiten sowohl im Bundestag als auch im Bundesrat erforderlich (was diesem wieder eine Vetoposition einräumt). Aus diesem Grund wurde für die Vor-

15 Kathrin Hämmerle, » >Niemand soll sagen können, er wäre nicht gehört worden ...: Betrachtungen zum Österreich- Konvent aus Sicht der Zivilgesellschaft" (2005) 34 Österreichische Zeitschrift für Politikwissenschaft 367 http://nbn-resolving.d e/urn:nbn:de:0168-ssoar-63490 (abgerufen am 31.01.2019).

16 Siehe Karl Kössler, "Streamlining Austria’s Federation: Comprehensive Reform after Nearly a Century? « (Constitution Net, 21. November 2018) www.constitution net.org/news/streamlining-austrias-federation-comprehensive-reform-after-nearlycentury (abgerufen am 31.01.2019). 
bereitung der Reform ein anderer Kontext gesucht. Beide Prinzipien, das Demokratie- und das Bundesstaatsprinzip, wurden in neuer Form vereint und eine ad hoc-Kommission zur Modernisierung der bundesstaatlichen Ordnung eingesetzt. In ihr waren Bundestag und Bundesrat paritätisch mit jeweils 16 Mitgliedern vertreten, entsprechend dem Modell des Vermittlungsausschusses. Sie war hochrangig besetzt, mit Ministerpräsidenten und führenden Politikern der im Bundestag vertretenen Parteien. Die Kommission wurde von Experten unterstützt und beraten. Sie kam ihrem Auftrag, »durch die Neuverteilung der Gesetzgebungskompetenzen das Gleichgewicht zwischen Bund und Ländern wieder her(zu)stellen «, im Wesentlichen nach, allerdings wurden die Themen Neugliederung und Finanzföderalismus von Beginn an ausgeklammert.

Die Beratungen und Arbeiten führten fast zu einem von allen Seiten akzeptierten Ergebnis, allerdings scheiterte die Verabschiedung eines Abschlussberichts kurz vor Ende des Mandats an politischen Differenzen. Nach den Wahlen im September 2005 nahm jedoch die neue Große Koalition wesentliche Teile der Vorschläge auf und setzte sie mit der Föderalismusrefom I im formellen Verfahren als Verfassungsänderung um.

Auch wenn keine deliberativen Partizipationselemente im eigentlichen Sinne (Bürgerbeteiligung) zu verzeichnen sind, ist die sog. Föderalismusreform ein interessanter Fall, da auch hier die institutionellen Verfahren durch ein ad hoc eingesetztes Organ ergänzt wurde, welches die eigentliche Reform und die formellen Verfahren vorbereiten sollte. Diese im Vorfeld angesiedelten Beratungen sollten eine effizientere und vertiefte Befassung mit den Reformthemen ermöglichen und parteipolitische Lagerkämpfe vermeiden helfen.

\section{Viel Diskussion und keine Reform: zur Lage in Italien}

In Italien haben jahrzehntelange Diskussionen über notwendige Verfassungsreformen vor allem zu zahlreichen akademischen Studien und Vorschlägen geführt. Die Lage und die Defizite sind daher klar erkannt; Einigkeit herrscht insbesondere über die Notwendigkeit den Entscheidungsprozess auf gesamtstaatlicher Ebene zu beschleunigen und dazu das perfekte Zweikammersystem umzugestalten sowie die vertikalen Beziehungen zwischen Staat, Regionen und Gemeinden neu zu ordnen (trotz oder gerade wegen der in diesem Bereich durch die Verfassungsreform von 2001 geschaffene Situation). In der Vergangenheit wurde auch mit besonderen Verfahren und bikameralen Ausschüssen im Parlament experimeniert. 
Unter den verschiedenen Ideen zur Vorbereitung von Verfassungsänderungen in den letzten Jahren sind insbesondere diejenigen eines Verfassungskonvents und die eines Zweikammer-Sonderverfahrens hervorzuheben. Die Idee eines Verfassungskonvents, bestehend jeweils zur Hälfte aus gewählten Abgeordneten und aus ernannten Experten, wurde jedoch angesichts massiver Kritik wegen der unterschiedlichen Legitimation dieser beiden Gruppen verworfen. Auch die Pläne für ein besonderes Verfahren mit einem Sonderausschuss von 42 Parlamentarierern aus beiden Kammern (anstelle der fast 1.000 Senatoren und Abgeordneten), die einen Vorschlag erarbeiten sollten, welcher dann von einer besonderen Mehrheit im Parlament verabschiedet und durch ein Referendum angenommen werden sollte, wurden letztendlich wegen der eindeutigen Unvereinbarkeit mit dem in Art. 138 itVerf geregelten Verfahren zur Verfassungsänderung aufgegeben. Es sollte kein Präzedenzfall zur Umgehung dieses Artikels geschaffen werden. Jedoch wurden neue Elemente zur Vorbereitung zukünftiger Reformen genutzt. Während der Präsidentschaft Giorgio Napolitanos wurde von diesem ein 42 Mitglieder starker Expertenausschuss als Konsultativorgan des Präsidenten eingerichtet, welcher Vorschläge für das Parlament erarbeiten und koordinieren sollte.

Parallel dazu wurde von der Regierung Letta ein Konsultationsprozess begonnen, welcher die italienischen Bürger erreichen wollte. Es handelte sich um einen strukturierten Prozess, nicht nur um eine Online-Befragung. Auf einer Internetplattform wurden zahlreiche Fragen zu Einzelheiten einer möglichen Verfassungsreform gestellt, z.B. zur Regierungsform, zur direkten Demokratie, zur territorialen Gliederung und Selbstverwaltung. Zwar hatte diese Konsultation wenig Echo in den Medien, trotzdem nahmen an ihr mehr als 200000 Bürger teil, obwohl eine Registrierung notwendig war, um Missbrauch zu verhindern, insbesondere Mehrfachantworten durch ein und dieselbe Person. Sämtliche Reformprioritäten wurden von den teilnehmenden Bürgern bestätigt; allerdings zeigt der Abschlussbericht auch eine Mehrheit gegen den von der Regierung geplanten Abbau regionaler Kompetenzen. ${ }^{17}$

In der Folge geschah jedoch nicht viel mit den Ergebnissen. Die neue Regierung von Matteo Renzi, welche im Februar 2014 Gianni Letta ablöste, gab die Idee eines besonderen Verfassungsgesetzes zur Einführung eines speziellen Verfassungsänderungsverfahrens auf und begann ohne weitere

17 Vgl. die für die Konsultation genutzte Internetplattform »partecipa!« (»beteilige dich!«), auf der auch der Abschlussbericht der Konsultation veröffentlicht worden war (www.partecipa.gov.it). 
Vorbereitung oder Konsultationen das parlamentarische Verfahren zur Verfassungsänderung mit einem sehr umfangreichen Änderungsentwurf. Nach langen Beratungen und Abstimmungen in beiden Kammern wurde das umfangreiche Verfassungsänderungsvorhaben schließlich verabschiedet und die Regierung Renzi war selbst treibende Kraft für ein - eigentlich nicht notwendiges - Verfassungsreferendum. Dies war ein politischer Fehler, da die Diskussion über die umfassende Verfassungsreform das Land spaltete, zur Möglichkeit der persönlichen Abrechnung mit Matteo Renzi wurde und die Personalisierung seinen Gegnern die Möglichkeit bot, sich gegen ihn zu verbünden. Die Verfassungsreform fiel entsprechend im Referendum vom 4. Dezember 2016 durch; Matteo Renzi trat zurück.

Der Grundsatz umfassender öffentlicher Konsultation ist jedoch mittlerweile in Italien etabliert und die Internetplattform wird weiter regelmäßig zur Online-Konsultation der Bürger für andere Gesetzgebungsvorgaben genutzt.

\section{Island: Ein völlig neues, mehrstufiges Modell für Bürgerbeteiligung?}

Nach den dramatischen Folgen der Wirtschafts- und Finanzkrise von 2008, welche für Island einen Bankenkollaps bedeutete, wurde allgemein eine grundlegende Erneuerung befürwortet. Dies schloss Forderungen nach einer neuen Verfassung ausdrücklich ein. 2009 wurde eine Nationalversammlung für ein nationales »Brainstorming « abgehalten. ${ }^{18}$ Auf dieser ging es vor allem um Grundwerte sowie um besonders wichtige Einzelfragen wie beispielsweise Ausbildung, Wirtschaft und Umwelt. Außerdem wurden Möglichkeiten einer umfassenden Verfassungsreform mit deliberativen Partizipationsformen diskutiert.

Dies wurde von der isländischen Regierung akzeptiert und im Juni 2010 wurde ein Gesetz zur Einberufung einer Verfassungsversammlung verabschiedet (Constitutional Assembly Act). Demnach sollte ein Verfassungsrat

18 Silvia Suteu, "Constitutional Conventions in the Digital Era: Lessons from Iceland and Ireland «, in (2015) 3 Boston College International and Comparative Law Review 251; Björg Thorarensen, »The People's Contribution to Constitutional Changes: Writing, Advising or Approving? - Lessons from Iceland «, in Xenophon Contiades and Alkemene Fotiadou (Hrsg.), Participatory Constitutional Change, (Routledge, 2017), 103ff; Eirikur Bergmann, »Participatory Constitutional Deliberation in the Wake of Crisis: The Case of Iceland «, in Min Reuchamps and Jane Suiter (Hrsg.), Constitutional Deliberative Democracy in Europe (ECPR Press, 2017), $15 \mathrm{ff}$. 
gewählt werden, nachdem eine Crowdsourcing-Versammlung zu Verfassungsfragen abgehalten worden war, als ein Tagesereignis für Bürgerbeteiligung, das von einem Verfassungsausschuss von sieben Experten vorbereitet werden sollte. Am 6. November 2010 fand die erste Phase statt: Unter Ausschluss von Mitgliedern politischer Parteien nahmen insgesamt 950 durch die Zufallsmethode ausgewählte Isländer an insgesamt 128 verschiedenen Runden Tischen teil. Auf diese Weise wurden acht Hauptthemen für die isländische Verfassung ermittelt, welche als Grundlage für die Beratungen und Arbeiten im Verfassungsrat vorgegeben wurden.

Am 27. November 2010 wollte man mit der Wahl der 25 Mitglieder des Verfassungsrates in die zweite Phase eintreten. 522 Isländer hatten sich selbst als Kandidaten zur Wahl gestellt. Wieder waren Abgeordnete ausgeschlossen. Gewählt wurde mit einem Verhältniswahlsystem (single transferable vote system); allerdings war die Wahlbeteiligung mit nur $27 \%$ sehr enttäuschend. Dies entspricht etwa der Hälfte der gewöhnlichen Wahlbeteiligung. Zudem kam es zu Problemen beim Wahlvorgang und es wurden Unregelmäßigkeiten behauptet, weswegen der Oberste Gerichtshof schließlich die Wahlen annullierte.

Daraufhin wurde der Verfassungsrat vom Parlament ernannt und begann seine Arbeit im April 2011 auf der Grundlage der Vorarbeiten der Verfassung. Es wurde großer Wert auf die Transparenz der Arbeiten gelegt: auf einer interaktiven Webseite wurden alle Entwürfe sowie Kommentare und Vorschläge dazu veröffentlicht. Jeder Bürger hatte das Recht, online Änderungen vorzuschlagen und am Text mitzuschreiben. Nach vier Monaten sandte der Verfassungsrat im Juli 2011 einen Verfassungsentwurf an das Parlament, nachdem dieser im Konsens verabschiedet worden war. Der Entwurf wurde danach von internationalen Experten sowie der Venedig-Kommission des Europarates begutachtet und von verschiedenen Seiten kritisiert. Anschließend kam es zunächst für Monate zu einem Stillstand im isländischen Parlament.

Schließlich wurde am 20. Oktober 2012 ein konsultatives Referendum über den Verfassungsentwurf abgehalten, bei dem insgesamt sechs Fragen zur Abstimmung gestellt wurden: die Bürger konnten sowohl über den Gesamtentwurf, als auch über fünf inhaltliche Hauptfragen abstimmen (ob natürliche Resourcen staatliches Eigentum sein sollten; über eine Bestimmung zur Staatskirche; über die Wahl bestimmter Individuen in den Althing; über das gleiche Stimmengewicht in allen Landesteilen bei Wahlen; über das Recht einer bestimmten Anzahl von Wählern Referenda zu beantragen). Die Wahlbeteiligung lag immerhin bei 49\% und alle Fragen wurden mit einer deutlichen Mehrheit positiv beantwortet. 
Allerdings kam es nach dem eindeutigen Ergebnis des nicht bindenden Referendums zu Streitigkeiten zwischen den politischen Parteien über die Umsetzung. Nach vielen Debatten kam es nie zu einer Endabstimmung darüber. Stattdessen wurde 2013 ein anderer Gesetzentwurf eingebracht, mit dem die Diskussion und die Entscheidung bis auf die Zeit nach den Wahlen im selben Jahr verschoben wurde, ${ }^{19}$ allerdings mit dem Zusatz, dass eine Verfassungsreform spätestens bis April 2017 erfolgen, vom Parlament mit Zweidrittelmehrheit verabschiedet und durch ein Referendum (mit mindestens 40\% Wahlbeteiligung als Gültigkeitserfordernis) bestätigt werden sollte. Damit hatte das Parlament die Hoheit über den Verfassungsänderungsprozess wiedererlangt.

Interessant sind im Fall Islands vor allem das Wechselspiel deliberativer Partizipationsformen mit den Institutionen der repräsentativen Demokratie, welche letztlich die Oberhand behielt sowie das Eingreifen des Obersten Gerichtshofes und die Wirkungen der Gutachten der Venedigkommission. Allerdings war die vorausgegangene Phase der Bürgerbeteiligung als wichtiges Element des Gesamtprozesses eingeführt und erprobt und international viel beachtet worden. Zudem war die Notwendigkeit eines komplementären Prozesses anerkannt worden.

Wenn man lediglich das Ergebnis betrachtet, muss das isländische Experiment mit dem Verfassungskonvent von 2012 also als ein Fehlschlag betrachtet werden, da das erklärte Ziel, eine neue Verfassung zu verabschieden, nicht erreicht wurde. Trotzdem war es das erste Mal, dass eine vollständig neue Verfassung im Wege deliberativer Partizipationsformen erarbeitet werden sollte. Trotz des (vermutlich zu) ehrgeizigen Zieles einer Gesamtrevision und des Fehlschlags hat das Verfahren enormes Interesse geweckt und ein großes Echo in der Welt und in der wissenschaftlichen Diskussion ausgelöst, weshalb es als durchaus Auslöser eines neuen Trends in Verfassungsänderungsverfahren angesehen werden kann.

19 Die Venedig-Kommission des Europarates hatte empfohlen, weitere Detailarbeiten am Verfassungsentwurf erst nach den anstehenden Wahlen anzugehen. Die vorgesehenen vielen Möglichkeiten für die direkte Intervention der Bürger seien zwar zu begrüßen, könnten aber auch Risiken der Blockade und der Instabilität mit sich bringen. Zudem seien zahlreiche Bestimmungen des Entwurfs noch zu vage geblieben. 


\section{Konkrete Einzeländerungen: der irische Verfassungskonvent mit Bürgermebrheit}

In Irland wurden bereits im Wahlkampf 2011 Verfassungsänderungsvorschläge von verschiedenen Parteien vorgeschlagen und diskutiert. 2012 wurde dann ein Verfassungskonvent (Irish: An Coinbhinsiún ar an mBunreacht) eingerichtet, um die vorgeschlagenen Verfassungsänderungsvorschläge zu diskutieren. Seine Zusammensetzung war gemischt: Unter den 100 Mitgliedern waren als Vertreter der Politik neben dem Vorsitzenden weitere 29 Mitglieder des irischen Parlaments und vier Vertreter der politischen Parteien Nordirlands; ${ }^{20}$ zwei Drittel des Konvents waren jedoch durch das Zufallsprinzip ermittelte Bürger. Die 66 Bürger wurden von einer Marktforschungsgesellschaft durch einen Algorithmus ausgewählt, der einen repräsentativen Querschnitt der Wahlbevölkerungsstruktur im Hinblick auf Alter, Region und Geschlecht garantierte; ebenso wurden 66 Ersatzmitglieder ausgewählt. ${ }^{21}$

Acht Einzelthemen sollten im Verfassungskonvent behandelt werden, diese Liste wurde vom Konvent um weitere Themen ergänzt:

- Die Verkürzung der Amtszeit des Präsidenten auf fünf Jahre und die Zusammenlegung mit den Gemeinde- und Europawahlen;

- Die Herabsetzung des Wahlalters auf 17 Jahre;

- Die Reform des Wahlsystems zur Kammer (Dáil);

- Die Ermöglichung des Wahlrechts für im Ausland ansässige irische Staatsbürger bei den Präsidentenwahlen in irischen Botschaften oder durch ähnliche Massnahmen;

- Eine Verfassungsbestimmung über die gleichgeschlechtliche Ehe;

- Eine Verfassungsänderung zur Rolle der Frauen mit dem Ziel ihrer größeren Beteiligung im öffentlichen Leben;

- Größere Beteiligung von Frauen in der Politik;

- Die Streichung einer Verfassungsvorschrift über das Vergehen der Blasphemie;

20 Die 29 Plätze für irische Abgeordnete wurden unter den im Parlament vertretenen Fraktionen im Verhältnis zu ihrer Sitzanzahl in beiden Häusern aufgeteilt; Sinn Fein war mit einer gemeinsamen Delegation aus beiden Häusern vertreten. Von den sechs eingeladenen nordirischen Parteien lehnten die protestantischen Parteien Democratic Unionist Party und Ulster Unionist Party eine Teilnahme ab, da sie den Konvent als inner-irische Angelegenheit betrachteten.

21 Statt der vollständigen Postanschrift wurden nur Name und Postleitzahl der Bürger veröffentlicht, um Befürchtungen einer Einflussnahme von Lobbyisten und Interessengruppen vorzubeugen. 
Andere relevante Verfassungsänderungen, welche der Konvent empfehlen wollte (nach der Behandlung der vorgenannten Themen).

Die erste Sitzung war am 1. Dezember 2012, der Konvent tagte bis 31. März 2014. Die ersten beiden Themen wurden zuerst bearbeitet; die entsprechenden Empfehlungen sollten bereits zwei Monate nach dem ersten Treffen an die Regierung abgegeben werden. Die anderen Themen sollten bis November 2013 bearbeitet warden. Nach diesem Zeitpunkt wurden die Reform der Abgeordnetenkammer (Dáil) und wirtschaftliche, soziale und kulturelle Rechte als zwei weitere Themen vom Konvent selbst gewählt.

Der Konvent arbeitete an 10 Wochenenden für jeweils eineinhalb Tage. Die Arbeitsmethode sah für jede Sitzung drei unterschiedliche Abschnitte vor: zunächst stellten am Samstag Experten ihre zuvor versandten Gutachten vor, darauf folgten Debatten zwischen Gruppen von Befürwortern und Gegnern der Vorschläge zum jeweiligen Thema und abschließend offene Diskussionen an Runden Tischen mit Moderatoren. Die Diskussionen wurden aufgezeichnet. Am folgenden Sonntagmorgen reflektierten die Konventsmitglieder über die Diskussionsergebnisse des Vortages. Zum Abschluss wurde auf einem Stimmzettel abgestimmt, auf dem die Hauptargumente aufgelistet waren. Alle Sitzungen waren öffentlich, und konnten durch Streaming verfolgt werden.

Die irische Regierung war nicht zur Berücksichtigung der Änderungsvorschläge des Konvents verpflichtet. Sie versprach aber eine förmliche Antwort auf alle Empfehlungen sowie eine Debatte darüber im Parlament. Dies sollte der Verknüpfung zwischen deliberativen und repräsentativen Verfahrenselementen ebenso dienen, wie der Achtung der vom Konvent geleisteten Arbeit und der Stellung des Parlaments.

Bis Dezember 2015 hatte die irische Regierung auf sechs der neun Berichte des Konvents formell geantwortet. Zwei Konventsvorschläge wurden am 22. Mai 2015 in einem Verfassungsreferendum zur Abstimmung gestellt. Es ging um die Einführung der gleichgeschlechtlichen Ehe und um die Herabsetzung der Altersgrenze für die Wählbarkeit zum Präsidentenamt von 35 auf 21 Jahre. Während die gleichgeschlechtliche Ehe akzeptiert und damit eingeführt wurde, wurde die Herabsetzung der Altersgrenze von einer Mehrheit der Wähler abgelehnt.

Vor den Wahlen 2016 schlugen verschiedene Politiker Änderungen an der irischen Abtreibungsgesetzgebung vor, einschließlich der Abschaffung des verfassungsrechtlichen Abtreibungsverbotes im Achten Verfassungszusatz. Dazu wurde vor den Wahlen erneut eine Bürgerversammlung (Citi- 
zens $^{6}$ Assembly $)^{22}$ versprochen, welche nach den Wahlen auch gebildet wurde. Ohne die Beteiligung von Politikern sollte diese über einen längeren Zeitraum eine begrenzte Anzahl von wichtigen Themen bearbeiten. $\mathrm{Zu}$ diesen gehörten insbesondere das Abtreibungsverbot, die Dauer der Legislaturperiode sowie die Art und Weise der Abhaltung von Referenda (z.B. die Zusammenlegung verschiedener Themen auf einen Wahltag), obwohl der Bürgerkonvent nicht auf Verfassungsthemen beschränkt war. Die 2017 abgeschlossenen Arbeiten wurden im Parlament von einem Ausschuss weiter beraten. Am 25. Mai 2018 wurde ein Referendum zum Achten Verfassungszusatz durchgeführt, bei dem sich eine Zweidrittelmehrheit der Abstimmenden für eine Änderung aussprachen, welche es dem irischen Parlament nun ermöglicht, Schwangerschaftsabbrüche zu legalisieren. ${ }^{23}$

Irland ist ein Beispiel für gelungenen und zielführenden Einsatz deliberativer Partizipationsformen zur Änderung einzelner Vorschriften einer staatlichen Verfassung. Der Verfassungskonvent hatte diese als Beratungsorgan vorbereitet und einen komplexen Katalog von insgesamt etwa 40 verschiedenen Änderungsempfehlungen erarbeitet; diese sollten durch die Regierung umgesetzt werden. Von ihnen erforderten 20 Empfehlungen Verfassungsänderungen. Während einige noch unerledigt sind, wurde die Ehe gleichgeschlechtlicher Paare durch das Referendum im Mai 2015 ermöglicht; ein beachtliches Ergebnis in einer bekanntermaßen katholisch geprägten und konservativen Rechtsordnung. Die Regierung hat in Aussicht gestellt, dass weitere Empfehlungen 2018 und 2019 in einem Referendum zur Abstimmung gestellt werden.

\section{Neue Wege durch Partizipation? Schlussfolgerungen}

In der vergleichenden Untersuchung lassen sich zwei große Fallgruppen unterscheiden: in Österreich, Deutschland und Italien wurden dem eigentlichen Verfassungsänderungsverfahren ein Konvent, eine besonders zusammengesetzte Kommission oder ein Expertengremium vorgeschaltet, um

22 Laura Cahillane, »Delaying Tactics or Useful Deliberative Exercises? The Irish Citizens' Assembly and the Convention on the Constitution « (IACL-AIDC Blog, 3. Dezember 2018) blog-iacl-aidc.org/debate-the-citizens-assembly-in-ireland/ 2018/12/3/delaying-tactics-or-useful-deliberative-exercises-the-irish-citizens-assembly-and-the-convention-on-the-constitution (abgerufen am 31.01.2019).

23 Bei einer Wahlbeteiligung 64,3\%. Zur Abstimmung stand konkret die Frage, ob die folgende Ergänzung in Art. 40.3.3 eingeführt werden sollte: »Bestimmungen zum Schwangerschaftsabbruch können gesetzlich geregelt werden.« 
eine vertiefte und breitere Diskussion der Reforminhalte zu ermöglichen, bevor sich das Parlament mit ihnen befasst. Auch wenn die Ergebnisse dieser Gremien nicht unmittelbar verwendet wurden, hatten sie jedoch auf den Fortgang der danach folgenden förmlichen Verfahren und auf die Inhalte folgender (Teil-)Reformen entscheidenden oder zumindest großen Einfluss. Eine echte Einbeziehung von Bürgern fand jedoch in diesen Fällen nicht statt, mit Ausnahme Österreichs und deren Einbindung in die parlamentarische Kommission, deren Arbeit jedoch nicht von Erfolg gekrönt war. In Italien hat die Online-Konsultation zwar einige Impulse gegeben, diese haben jedoch keine besonders sichtbaren Auswirkungen auf den nachfolgenden Verfassunsgreformentwurf gezeitigt.

Demgegenüber sind die Instrumente, die in Irland und Island genutzt wurden, wesentlich innovativer, weitreichender und auch im Hinblick auf die Entscheidungen einflussreicher. Sie kommen damit den grundlegenden Intentionen der deliberativen und partizipativen Demokratie sehr viel näher und beweisen deren Anwendbarkeit und Potential auch auf der Verfassungsebene.

Die drei Modelle - direkte, repräsentative und deliberative Demokratie - kann man mit unterschiedlichen Fahrrädern vergleichen: das Hochrad des 19. Jahrhunderts, das heutige Standardfahrrad und ein Lastenfahrrad mit drei Rädern. Das Hochrad symbolisiert dabei die direkte Demokratie, das Verfassungsreferendum und die direkte Ausübung der Entscheidungsgewalt durch das Volk. Es war eine revolutionäre Erfindung bei seiner Einführung, ist aber langsam und schwerfällig und schwierig im Gleichgewicht zu halten. Dies und seine kurze Reichweite schränken seine universale Einsetzbarkeit ein, was der binären Logik des "Ja oder Nein« bei Referenden entspricht, die für Grundsatzentscheidungen geeignet sein mögen, jedoch kaum als alltägliches Entscheidungsinstrument und für differenzierte Entscheidungen eine vorgeschaltete Informationsund Diskussionsphase benötigen. Das Prinzip des Hochrades ist daher zugunsten einer deutlichen Verbesserung von Stabilität und Geschwindigkeit konzeptionell geändert worden. Dem Standardfahrrad von heute entspricht das Konzept der repräsentativen Demokratie. Sie wird auf alle Entscheidungsfragen angewendet, einschließlich der meisten Verfassungsänderungsverfahren. Die Änderungen am Konzept, wobei das Prinzip Fahrrad gleichbleibt, berücksichtigen auch den Pluralismus der modernen Gesellschaft in den Parlamenten, was durch die Variante des Damenfahrrades dargestellt werden kann, welches die Vertretung der bis dahin von der Politk ausgeschlossenen Frauen symbolisiert. Trotzdem ist in bestimmten Situationen, z.B. für Lastentransporte, eine besondere Weiterentwicklung möglich, beispielsweise durch Hinzufügen eines dritten Rades für 
noch mehr Sicherheit und Stabilität. Dies ließe sich mit deliberativen Partizipationsprozessen vergleichen, welche zur Vorbereitung grundlegender Entscheidungen eine umfassendere Vertiefung der Entscheidungsfaktoren und zur Steigerung der Akzeptanz und Nachhaltigkeit die Einbindung der Bürger in die Entscheidungsvorbereitung garantieren.

Der Vergleich ergibt für deliberative Partizipationsprozesse auf Verfassungsebene folgende Gemeinsamkeiten:

- Sie dienen der Vorbereitung der Entscheidungen in den Gremien im Vorfeld formeller Verfahren; im Idealfall bereits mittels Konsensbildung (dies ist jedoch nicht Voraussetzung).

- Beratung, Vertiefung von Sachfragen und Meinungsbildung außerhalb des Politikbetriebes bzw. der Institutionen der repräsentativen Demokratie stehen im Vordergrund.

- Wesentliches Kennzeichen ist die Bürgerbeteiligung, für die verschiedene Formen zur Verfügung stehen. Bei der Arbeitsmethode sind Transparenz und ein klares Regelwerk wesentlich.

- Ein großer Vorteil ist, dass deliberative Partizipationsmodelle nicht der Mehrheitslogik unterworfen sind (wie dies bei repäsentativer und direkter Demokratie der Fall ist). Da sie nicht unmittelbar mit den Entscheidungen verknüpft sind, sondern diese nur vorbereiten, sind sie auf das Erreichen nachhaltiger Lösungen ausgerichetet: Qualität und Akzeptanz stehen im Vordergrund. Daher ist Konsens häufig wünschenswert, wichtiger ist aber, dass jede Meinung zählt, unabhängig von zahlenmäßiger Stärke.

Bürgerbeteiligung und der Einsatz deliberativer Partizipationsformen erscheinen eher dafür geeignet, wichtige Einzelthemen durch vertiefte Diskussion für eine verfassungsändernde Entscheidung vorzubereiten, als für die Verfolgung ehrgeiziger Projekte einer umfassenden Revision der Verfassung. Der Vergleich verschiedener Fälle zeigt nämlich, dass die in den partizipativen Verfahren erzielten Ergebnisse häufig zunächst nicht unmittelbar verwirklicht werden, jedoch nicht selten zu einem späteren Zeitpunkt aufgegriffen und in ein traditionelles Verfahren eingeführt bzw. verabschiedet werden.

Im Verhältnis zur repräsentativen Demokratie handelt es sich nicht um alternative, sondern um komplementäre Formen. Vielleicht sind sie nicht unverzichtbar, sie versprechen aber durch die mit ihnen verbundenen Diskussionen und die Vorbereitung besonders wichtiger Entscheidungen in pluralistischen Gesellschaften in jedem Fall einen Qualitäts- und Nachhaltigkeitsgewinn für wichtige Reformen. Daher ist zu erwarten, dass auch 
Bürgerbeteiligung oder »Stimme des Volkes«?

auf der Ebene (gesamt)staatlicher Verfassungen weiter mit ihnen experimentiert wird. 
